\title{
Vectorial four-wave mixing field dynamics from individual excitonic transitions
}

\author{
Jacek Kasprzak and Wolfgang Langbein \\ School of Physics and Astronomy, Cardiff University, 5 the Parade, Cardiff CF24 3AA, United Kingdom
}

(Received 23 April 2008; revised manuscript received 4 June 2008; published 16 July 2008)

\begin{abstract}
Degenerate four-wave mixing (FWM) from individual localized excitonic transitions is measured using a dual-polarization extension of heterodyne spectral interferometry. A fine-structure splitting (FSS) of the localized excitons of $20-80 \mu \mathrm{eV}$ is observed, revealing a spatially anisotropic localization in the quantum well plane, preferentially oriented along the [110] direction. The ratio of the transition dipole moments of the FSS transitions is in the range $0.7-1.4$, with no significant correlation to the FSS. Retrieving both polarization components of the FWM in amplitude and phase we measure the time-resolved polarization state of the exciton after pulsed excitation, showing a coherent evolution oscillating between linear and virtually circular polarization. The polarization of the created FWM state is a result of the initial polarization state created by the first excitation pulse, its free evolution up to the arrival of the second pulse, and its conversion into the FWM controlled by the polarization of the second pulse.
\end{abstract}

DOI: 10.1103/PhysRevB.78.041103

PACS number(s): 78.47.Fg, 78.47.jj, 78.47.jm, 78.47.nj

With the recent development of the heterodyne spectral interferometry (HSI) technique, ${ }^{1}$ the measurement of the nonlinear coherent response, e.g., four-wave mixing (FWM), of individual localized two-level systems has become feasible. Standard FWM experiments ${ }^{2,3}$ rely on directional selection of the nonlinear optical response and therefore are not suited for emitters localized to subwavelength regions, like excitons confined in quantum dots. HSI overcomes this limitation by taking advantage of an effective frequency selection of the FWM signal, relying only on the time invariance of the system investigated. HSI has been used to determine broadening mechanisms and measure microscopic dephasing time within finite, inhomogenously broadened ensembles of excitons ${ }^{4}$ or transitions experiencing spectral wandering. ${ }^{5} \mathrm{By}$ applying spectral interferometry, ${ }^{6}$ the FWM is retrieved both in amplitude and phase. This permits not only to obtain complete information about the state vector on the Bloch sphere, but also allows the resonant observation of coherent control of excitons on ultrafast time scales. ${ }^{7}$ By performing delaytime dependent HSI and transforming-in analog to NMR techniques 8,9 - the data into a two-frequency representation $R^{-1,2}\left(\omega, \omega_{1}\right)$, one can observe and classify coherent coupling between individual localized excitons. ${ }^{1}$ Similar twodimensional techniques are used to investigate many-body interactions in quantum wells ${ }^{10}$ and vibrational and electronic coupling in molecules. ${ }^{11}$ HSI is therefore a powerful tool for coherent characterization, ultrafast coherent control, and manipulation of excitonic qbits.

In this work we extend the HSI technique to a dualpolarization scheme (DHSI), which allows us to simultaneously measure both polarization components of the field in phase and amplitude, completely determining the time or spectrally resolved polarization state of the FWM. A similar technique has been introduced ${ }^{12}$ and used for FWM from quantum wells earlier. ${ }^{13,14}$ We demonstrate DHSI for FWM of excitons localized in monolayer islands of a $5 \mathrm{~nm}$ thick growth interrupted GaAs/AlAs quantum well. Owing to their large lateral size of a few tens of nanometers, confined excitons exhibit high oscillator strengths and are well suited for measuring their coherent response. This sample has been previously investigated ${ }^{4,7,15}$ and its properties have been simulated. ${ }^{16}$ Here we focus on the fine-structure splitting (FSS) of the bright exciton into two nondegenerate, orthogonally linearly polarized transitions. ${ }^{17}$ The direction of the linear polarization is dictated by the in-plane anisotropy of the localizing structure. In nanostructures made of zincblende crystal structures grown in [001] direction, in which [001] is also the direction of strongest quantum confinement, the inplane directions of anisotropy are $[1 \overline{1} 0]$ and [110]. While the local confinement potential due to the random disorder of the growth interfaces results in a random component of the anisotropy, the observed anisotropy directions are typically aligned along $[1 \overline{1} 0]$ and $[110],{ }^{17-20}$ revealing an anisotropic interface roughness. This is generally attributed to different surface mobilities of the group III element along these two directions, induced by the surface reconstruction during growth. We report here on transitions having their FSS preferentially along these directions. Due to valence-band mixing and elongation of the exciton center-of-mass wave function, the degeneracy of the optical dipole moment $\mu$ of the two transitions is also lifted, ${ }^{21,22}$ so that in general $\mu_{[110]}$ $\neq \mu_{[110]}$. Using FWM the transition dipole moments $\mu$ can be measured using the FWM intensity $I_{\mathrm{F}} \propto \mu^{8}$. By simultaneously detecting the FWM signal along [110] and [1 10$]$ directions, the relative phase between both components is obtained, which allows to fully describe the time-dependent polarization state, not accessible using photoluminescence (PL). Moreover, PL is influenced by the capture rate, preventing the measurement of transition dipole moments.

Our experiments are based on the setup described in Refs. 1 and 15. A pair of spectrally shaped optical pulse trains $P_{1,2}$, with a controlled relative time delay $\tau$, is frequency up shifted by $\Omega_{1}$ and $\Omega_{2}$ using acousto-optical modulators (AOMs). These "phase cycled" excitation fields are recombined into the same spatial mode and focused onto the sample surface by a microscope objective of 0.85 NA. The objective is mounted on a $x-y-z$ translation stage inside a helium immersion cryostat. The emitted FWM field is retrieved by detecting the interference with a reference pulse train at the FWM frequency $\Omega_{\mathrm{F}}=2 \Omega_{2}-\Omega_{1}$. Using a defined time ordering of reference and signal fields, we perform 


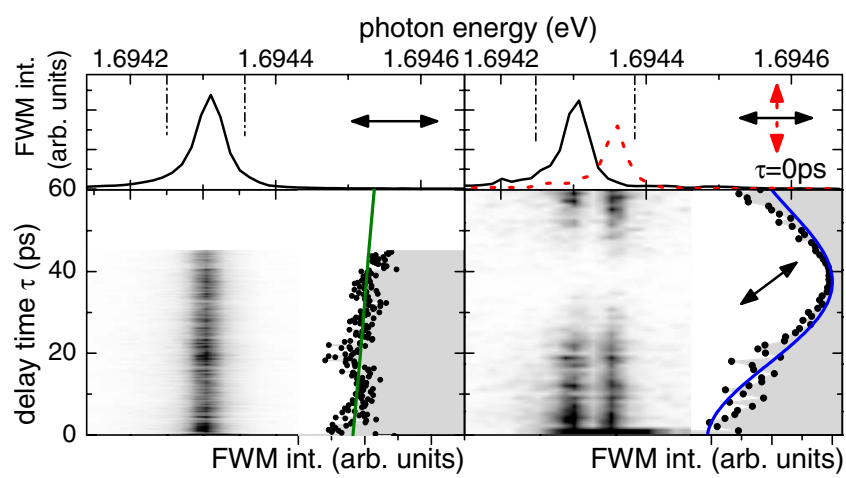

FIG. 1. (Color online) Spectrally resolved FWM intensity $I_{\mathrm{F}}(\tau, \omega)$ versus delay time $\tau$ from a homogenously broadened localized exciton transition. Left: $(\rightarrow \longrightarrow \rightarrow)$ polarization. Bottom: Linear gray scale plot from white (zero) to black, and resonant FWM $I_{\mathrm{F}}\left(\tau, \omega_{\mathrm{X}}\right)$ with fitted exponential decay (solid). Top: $I_{\mathrm{F}}(0, \omega)$. Right bottom: as left, but $\left(\nearrow \nearrow \nearrow\right.$ ) polarization. Fit of $I_{\mathrm{F}}\left(\tau, \omega_{\mathrm{X}}\right)$ (see

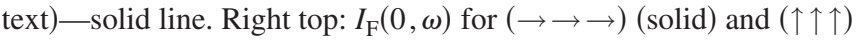
(dotted) polarization.

spectral interferometry to obtain the spectrally- or timeresolved FWM field in amplitude and phase. The polarization states of the excitation and reference fields were controlled by $\lambda / 2$ and $\lambda / 4$ wave plates. The dual-polarization detection is realized by a birefringent calcite plate cut at $45^{\circ}$ in front of the spectrometer input slit, displacing the foci of the $[1 \overline{1} 0]$ and [110] linear polarization components by about $120 \mu \mathrm{m}$ along the slit. Their spectra are measured individually in vertically displaced regions of the charge coupled device detector. In the following we will indicate the polarization of $P_{1}, P_{2}$, and detection by a triplet $(\rightarrow \nearrow \sigma)$, where the individual symbols are arrows for linear polarization along its direction (horizontal for [1 $\overline{1} 0]$ ), and $\sigma$ for circular polarization, respectively. Dual polarization detection is indicated as + . The $P_{1}$ beam has a fluence in the focus at the sample of $\sim 1 \mu \mathrm{J} / \mathrm{cm}^{2}$ per pulse of $\sim 200$ fs duration, and the relative power of $P_{1}, P_{2}$, and reference is 1:2:5. All data were taken at a sample temperature of $T=7 \mathrm{~K}$. When frequencies are given in energy units, they are multiplied by $\hbar$.

The averaged PL spectrum confocally excited over a region of $(4 \times 5) \mu \mathrm{m}^{2}$ size is presented in Fig. 2(a). One can observe emission from various localized transitions and from a high density of states at the high energy side originating from the next monolayer thickness. The fractional monolayer thickness of the selected sample region leads to a low spectral and spatial density of localized states, ${ }^{16}$ enabling us to find a region dominated by a single transition within the focal area both in PL and FWM [see black peak in Fig. 2(a)]. This transition is investigated in the following. The spectrally resolved FWM intensity for $(\rightarrow \rightarrow \rightarrow)$ polarization, and its delay-time dependence is shown in the left panel of Fig. 1. We have verified that the spectral wandering of this transition is not significant using the time-resolved FWM data $I_{\mathrm{F}}(t, \tau)$, which shows no sign of photon echo formation. ${ }^{4}$ From the measured linewidth of $\sim 40 \mu \mathrm{eV}$ FWHM, a homogenous linewidth of $\gamma=(7 \pm 4) \mu \mathrm{eV}$ FWHM is deduced taking into account the spectral response of the setup described by a Voigt profile of $22 \mu \mathrm{eV}$ Gaussian and $9 \mu \mathrm{eV}$

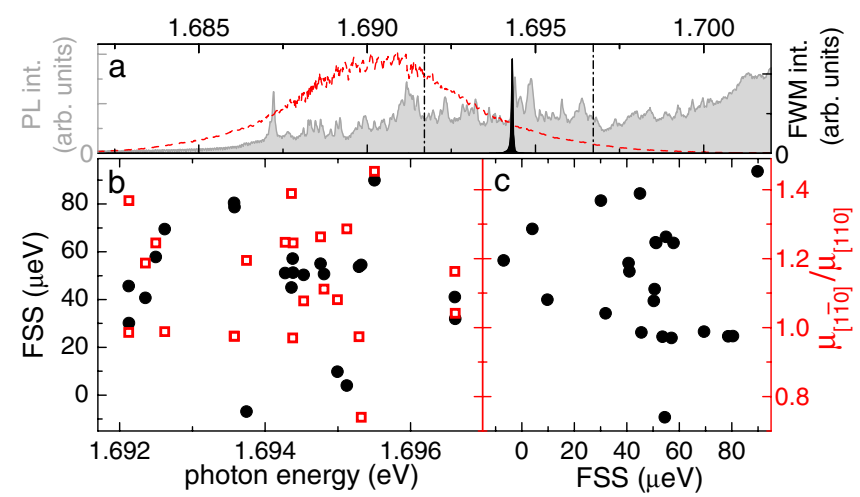

FIG. 2. (Color online) (a) FWM intensity of the investigated transition (black peak), averaged PL spectrum (gray area) nonresonantly excited at $1.69 \mathrm{eV}$ measured from a sample region of (4 $\times 5) \mu \mathrm{m}^{2}$ size around the probed transition, FWM excitation spectrum ( $\sim 200$ fs long pulse, dashed line), spectral region in (b) is marked by vertical dashed-dotted lines. (b) FSS energies $\Delta_{\mathrm{FS}}$ (black circles) and corresponding dipole moment ratios $\mu_{[1 \overline{10}]} / \mu_{[110]}$ (open squares) as a function of $\omega_{[1 \overline{10}]}$ (c) $\mu_{[1 \overline{10}]} / \mu_{[110]}$ as a function of $\Delta_{\mathrm{FS}}$.

Lorentzian width. ${ }^{23}$ This linewidth is in agreement with the polarization decay time $T_{2}=(400 \pm 100)$ ps deduced from the decay dynamics ${ }^{24}$ averaged over the spectral region marked by dashed-dotted lines in Fig. 1. Such a long dephasing time is likely to be due to radiative decay. ${ }^{16}$ We find that in the present experiment using $200 \mathrm{fs}$ pulses [see red dashed line in Fig. 2(a)], the measured $\gamma$ increases approximately linearly with the excitation power, by about $\sim 5 \mu \mathrm{eV} /\left(\mu \mathrm{J} / \mathrm{cm}^{2}\right)$, significantly more than observed previously in experiments using 2 ps long pulses. ${ }^{7}$ This can be understood by the spectrally wider excitation, which is more prone to excite extended excitonic states, resulting in an effect similar to excitation induced dephasing. ${ }^{25}$

For $(\uparrow \uparrow \uparrow)$ polarization, we find a spectrally shifted response (Fig. 1 top right). From the missing cross-talk between the two transitions in the FWM spectra, we can conclude that they are polarized to within $\sim 10^{\circ}$ along $[1 \overline{1} 0]$ and [110], respectively. The FSS of this transition is measured to be $\Delta_{\mathrm{FS}}=\omega_{[110]}-\omega_{[110]}=(54 \pm 2) \mu \mathrm{eV}$. From the FWM intensity ratio of the two transitions we can determine their relative oscillator strength to be $\mu_{[1 \overline{1} 0]} / \mu_{[110]}=1.069 \pm 0.005$. In order to excite an excitonic wave packet, we use $(\nearrow \nearrow \nearrow)$ polarization, for which the two transitions are excited with approximately equal strength. In this case, the first-order coherence created by $P_{1}$ has a time-dependent polarization direction, oscillating from $\nearrow$ to $\sigma^{+}$to $\nwarrow$ to $\sigma^{-}$to $\nearrow$, so that the density grating amplitude $\propto \exp \left(-\tau / T_{2}\right) \cos \left(\Delta_{\mathrm{FS}} \tau / 2\right)$ created by the $\nearrow$ polarized $P_{2}$ is oscillating. ${ }^{20}$ The resulting oscillation in the FWM intensity $I(\tau) \propto \exp \left(-2 \tau / T_{2}\right)[1$ $\left.+\cos \left(\Delta_{\mathrm{FS}} \tau\right)\right]$ is observed in the delay scan $I_{\mathrm{F}}(\omega, \tau)$ shown in Fig. 1 bottom right. The quantum beat, which occurs equally on both resonances, is well described by this prediction with $\Delta_{\mathrm{FS}}=(55 \pm 3) \mu \mathrm{eV}$ (see blue solid line), in agreement with the splitting measured in the FWM spectra.

In order to gain a statistical insight into distribution of FSS energies and their relation to dipole moment ratios between polarization components, we have probed a fixed sample area of $(4 \times 5) \mu \mathrm{m}^{2}$ and picked up 22 localized tran- 
sitions exhibiting strong FWM and significant $\Delta_{\mathrm{FS}}$ $>20 \mu \mathrm{eV}$. The resulting distribution of FSS energies shows $\left|\Delta_{\mathrm{FS}}\right|$ up to $80 \mu \mathrm{eV}$ with an average of $\sim 40 \mu \mathrm{eV}$. Furthermore, we find that in nearly all cases $\Delta_{\mathrm{FS}}>0$ and $\mu_{[1 \overline{10}]} / \mu_{[110]}>1$, i.e., the $[1 \overline{1} 0]$ polarized transition is at lower energy and has the larger oscillator strength. This is due to the anisotropic localization, ${ }^{17,18,26}$ with the longer extension along the $[1 \overline{1} 0]$ direction. The fraction of transitions which are aligned along [1] 0$]$ within $\pm 15^{\circ}$ (resulting in a cross-talk in the FWM intensity below $10 \%$ ) is $\sim 70 \%$. The values of $\Delta_{\mathrm{FS}}$ and $\mu_{[1 \overline{10}]} / \mu_{[110]}$ for these transitions as function of their transition energy $\omega_{[1 \overline{10}]}$ and $\mu_{[1 \overline{10}]} / \mu_{[110]}$ as a function of $\Delta_{\mathrm{FS}}$ are shown in Figs. 2(b) and 2(c). No significant correlation between any of these quantities is visible.

We now turn to the dual-polarization detection of the FWM field in amplitude and phase, with which the timeresolved polarization state of an exciton can be fully determined. To do so we use a $\nearrow$ polarized reference field, having equal amplitude and zero phase shift between the two detected polarization components along $\rightarrow$ and $\uparrow$. To compensate for different sensitivities and phase shifts of the two polarizations in the detection (mainly due to the grating in the spectrometer and the birefringence of the AOM), we use the instantaneous FWM at zero delay, i.e., the field $\overrightarrow{\mathcal{E}}_{\mathrm{F}}(\tau$ $=0, t=0)$, which is dominated by the isotropic GaAs bulk response. $\overrightarrow{\mathcal{E}}_{\mathrm{F}}(0,0)$ is therefore expected to be polarized along $\nearrow$ for $(\nearrow \nearrow+)$ configuration. Any deviation from this polarization measured in the DHSI is compensated by normalizing and phase shifting the $\uparrow$ polarized field component $\mathcal{E}_{\mathrm{F} \uparrow}$ so that $\mathcal{E}_{\mathrm{F} \rightarrow}(0,0)=\mathcal{E}_{\mathrm{F} \uparrow}(0,0)$. This instantaneous FWM corresponds to a spectrally broad background, which we removed from $\overrightarrow{\mathcal{E}}_{\mathrm{F}}$ by setting the field for $t<0.25 \mathrm{ps}$ to zero in the FWM data analyzed in the following. To suppress noise and weaker transitions spectrally distinct from the main transition, we transform the data to the spectral domain, filter with a Gaussian centered on the transition energy (see insets in Fig. 3), and transform the result back to the time domain. The intensity full width at half maximum (FWHM) $\Delta_{\omega}$ of the Gaussian filter results in a convolution of the time-domain data with a Gaussian of FWHM $\Delta_{t}=\left[4 \ln (2) \Delta_{\omega}\right]^{-1}$, limiting the time resolution of the resulting data. The result of this procedure is shown in Fig. 3 for $(\nearrow \nearrow+)$ and $(\sigma \sigma+)$ configuration. The relative phase between both polarization components [Figs. 3(a) and 3(c)] shows a linear slope proportional to the FSS. The FWM field amplitudes [Figs. 3(b) and $3(\mathrm{~d})]$ are decaying with time, and show some additional beating dynamics. The finite spectral resolution of the spectrometer used in the experiment results in a response in the time-resolved data which is decreasing with increasing time $t$ [see dashed-dotted line in Fig. 3(b)] which is relevant but not detrimental.

An intuitive representation of the polarization state dynamics is given by the angles of the polarization ellipse ${ }^{27}$ $(\psi, \chi)$ (marked on the Poincare sphere in the inset of Fig. 4(b), which are the azimuth angle of the ellipse and the arcus tangens of the ellipticity, respectively. They are connected with the Stokes parameters by $2 \psi=\arctan (U / Q), 2 \chi$ $=\arctan \left(V / \sqrt{Q^{2}+U^{2}}\right)$, with $Q=\left|\mathcal{E}_{\rightarrow}\right|^{2}-\left|\mathcal{E}_{\uparrow}\right|^{2}, \quad U=2 \Re\left(\mathcal{E}_{\rightarrow} \mathcal{E}_{\uparrow}^{\star}\right)$,

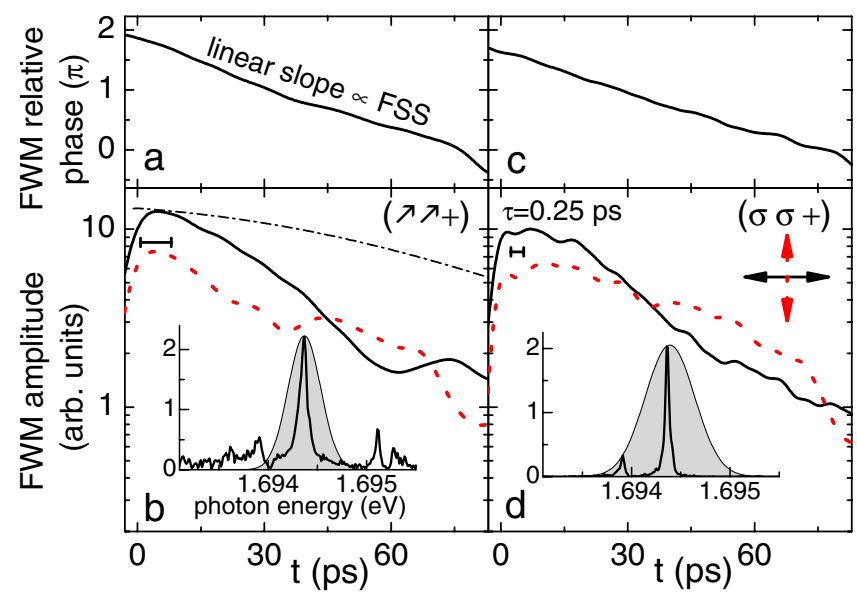

FIG. 3. (Color online) Time and polarization resolved FWM field $\overrightarrow{\mathcal{E}}_{\mathrm{F}}(0.25 \mathrm{ps}, t)$ from a FSS exciton transition. (a), (b) $(\nearrow \nearrow+)$ and $(\mathrm{c}),(\mathrm{d})(\sigma \sigma+)$ configuration. (b), (d) amplitude and (a), (c) relative phase of the field components $\mathcal{E}_{\mathrm{F} \rightarrow}$ (solid), $\mathcal{E}_{\mathrm{F} \uparrow}$ (dotted). The time resolution of the data due to the spectral filtering $\Delta_{t}$ is 7.5 ps [(a), (b)] and 2.9 ps [(c), (d)]—marked by horizontal bars. Dash-dotted line in (b): system response of the HSI setup. Insets: FWM amplitude (black) and spectral filter function (gray).

$V=2 \mathfrak{I}\left(E_{\rightarrow} \mathcal{E}_{\uparrow}^{\star}\right)$, and $\mathfrak{R}$ and $\mathfrak{I}$ real and imaginary part, respectively. The representation of the FWM data in $(2 \psi, 2 \chi)$ is given in Figs. 4(a) and 4(b). In the evolution of $\psi$ we observe a continuous clockwise rotation of the polarization ellipse, and a temporal oscillation of $\chi$ showing the evolution between linear and nearly circular polarization. This behavior is illustrated in Fig. 4(c), where the polarization ellipses for different times after collinear excitation are depicted. Here

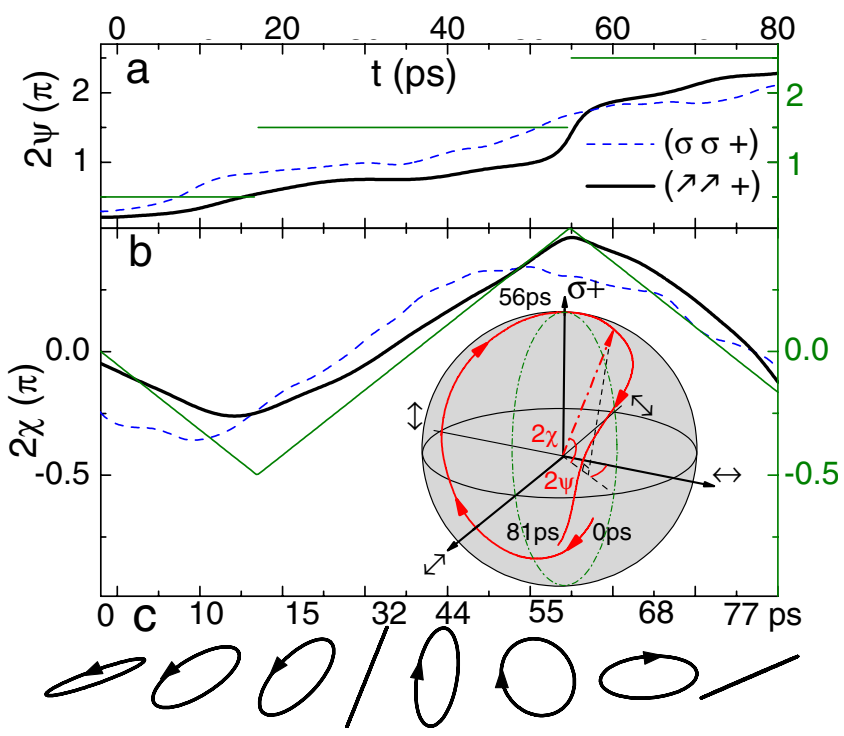

FIG. 4. (Color online) Time-resolved polarization ellipse of the FWM field, expressed by the Stokes angles $\psi$ and $\chi$. The solid and dashed curves correspond to $(\nearrow \nearrow+)$ and $(\sigma \sigma+)$ configuration. Thin lines: calculated dynamics for a pair of linearly polarized FSS transitions of equal amplitude. Inset: Poincaré sphere with marked $2 \psi$ and $2 \chi$ angles. Solid line: time path of the polarization state for $(\nearrow \nearrow+)$. (c) Polarization ellipses at different times for $(\nearrow \nearrow+)$. 
we directly observe evolution from initially almost linear polarization, through the elliptical polarization state, to rotated linear polarization, toward elliptical polarization of the opposite sign, and finally back to the initial linear polarization direction. Another convenient representation of the measured time-resolved polarization state is presented in the inset of Fig. 4, where the time path of the Stokes vector over the Poincaré sphere is depicted for $(\nearrow \nearrow+)$. The dynamics of an ideal pair of coupled oscillators, i.e., having the same field amplitudes (green thin lines in Fig. 4) is showing a qualitatively similar dynamics, with the deviations due to the different initial amplitudes and the residual noise in the experimental data. The time range of the experiment is limited to about 80 ps due to the radiative decay and the limited spectral resolution of our setup [see Figs. 3(b) and 3(d)]. Because only the coherent FWM emission is measured, the Stokes vector remains on the sphere surface. Finally, comparing the polarization dynamics of $(\nearrow \nearrow+)$ and $(\sigma \sigma+)$ con- figuration, the main difference is a time shift, as predicted from the different initial phase, zero and $\pi / 2$, between the FSS states.

In conclusion, we have extended the HSI technique to a dual-polarization detection scheme (DHSI). By retrieving the vectorial FWM field emitted by a single localized finestructure split exciton, we have completely measured its time-resolved polarization dynamics. We have confirmed that the fine-structure splitting introduces an state evolution in agreement with the theory for two linearly polarized coupled excitonic transitions. DHSI is not limited to the particular system studied here, but can be employed to describe the coherent evolution of any set of localized optical transitions.

We acknowledge support by the UK EPSRC under Contract No. EP/D025303/1, and Brian Patton for discussions and contributions to the experimental setup.
${ }^{1}$ W. Langbein and B. Patton, Opt. Lett. 31, 1151 (2006).

${ }^{2}$ J. Shah, Ultrafast Spectroscopy of Semiconductors and Semiconductor Nanostructures (Springer, Berlin, 1996), Chap. 2.

${ }^{3}$ S. Mukamel, Principles of Nonlinear Optical Spectroscopy (Oxford University Press, New York, 1999).

${ }^{4}$ W. Langbein and B. Patton, Phys. Rev. Lett. 95, 017403 (2005).

${ }^{5}$ B. Patton, W. Langbein, U. Woggon, L. Maingault, and H. Mariette, Phys. Rev. B 73, 235354 (2006).

${ }^{6}$ L. Lepetit, G. Chériaux, and M. Joffre, J. Opt. Soc. Am. B 12, 2467 (1995).

${ }^{7}$ B. Patton, U. Woggon, and W. Langbein, Phys. Rev. Lett. 95, 266401 (2005).

${ }^{8}$ J. Keeler, Understanding NMR Spectroscopy (Wiley, Chichester, 2005).

${ }^{9}$ R. Ernst, G. Bodenhausen, and A. Wokaun, Principles of Nuclear Magnetic Resonance in One and Two Dimensions (Oxford Science, New York, 1987).

${ }^{10}$ T. Zhang, I. Kuznetsova, T. Meier, X. Li, R. P. Mirin, P. Thomas, and S. T. Cundiff, Proc. Natl. Acad. Sci. U.S.A. 104, 14227 (2007).

${ }^{11}$ R. M. Hochstrasser, Proc. Natl. Acad. Sci. U.S.A. 104, 14190 (2007).

${ }^{12}$ W. J. Walecki, D. N. Fittinghoff, A. L. Smirl, and R. Trebino, Opt. Lett. 22, 81 (1997).

${ }^{13}$ O. Buccafusca, X. Chen, W. J. Walecki, and A. L. Smirl, J. Opt. Soc. Am. B 15, 1218 (1998).

${ }^{14}$ A. L. Smirl, M. J. Stevens, X. Chen, and O. Buccafusca, Phys. Rev. B 60, 8267 (1999).
${ }^{15}$ W. Langbein and B. Patton, J. Phys.: Condens. Matter 19, 295203 (2007).

${ }^{16}$ V. Savona and W. Langbein, Phys. Rev. B 74, 075311 (2006).

${ }^{17}$ D. Gammon, E. S. Snow, B. V. Shanabrook, D. S. Katzer, and D. Park, Phys. Rev. Lett. 76, 3005 (1996).

${ }^{18}$ W. Langbein, R. Zimmermann, E. Runge, and J. M. Hvam, Phys. Status Solidi B 221, 349 (2000).

${ }^{19}$ M. Bayer, G. Ortner, O. Stern, A. Kuther, A. A. Gorbunov, A. Forchel, P. Hawrylak, S. Fafard, K. Hinzer, T. L. Reinecke, S. N. Walck, J. P. Reithmaier, F. Klopf, and F. Schäfer, Phys. Rev. B 65, 195315 (2002).

${ }^{20}$ W. Langbein, P. Borri, U. Woggon, V. Stavarache, D. Reuter, and A. D. Wieck, Phys. Rev. B 69, 161301(R) (2004).

${ }^{21}$ W. Langbein, P. Borri, U. Woggon, V. Stavarache, D. Reuter, and A. D. Wieck, Phys. Rev. B 70, 033301 (2004).

${ }^{22}$ A. Muller, Q. Q. Wang, P. Bianucci, C. K. Shih, and Q. K. Xue, Appl. Phys. Lett. 84, 981 (2004).

${ }^{23}$ G. Kocherscheidt, W. Langbein, G. Mannarini, and R. Zimmermann, Phys. Rev. B 66, 161314(R) (2002).

${ }^{24}$ The error is limited by the spatial drift of the setup during the experiment.

${ }^{25}$ A. Honold, L. Schultheis, J. Kuhl, and C. W. Tu, Phys. Rev. B 40, 6442 (1989).

${ }^{26}$ S. Goupalov, E. L. Ivchenko, and A. V. Kavokin, Superlattices Microstruct. 23, 1205 (1998).

${ }^{27}$ M. Born and E. Wolf, Principles of Optics (Cambridge University Press, Cambridge, UK, 1999), Chap. 1, p. 10. 